\title{
EL CONJUNTO MÍNIMO BÁSICO DE DATOS AL ALTA HOSPITALARIA COMO FUENTE DE INFORMACIÓN PARA EL ESTUDIO DE LAS ANOMALÍAS CONGÉNITAS
}

\author{
Nieves Alba Moratilla (1), Ana M. García García (2) y Fernando G. Benavides (3) \\ (1) Unidad de Salud Laboral. Centro de Salud Pública de Valencia. \\ (2) Departamento de Medicina Preventiva y Salud Pública, Bromatología, Toxicología y Medicina Legal. Univer- \\ sitat de València. \\ (3) Departamento de Ciencias de la Salud. Universidad Pompeu Fabra.
}

\section{RESUMEN}

Fundamento: El objetivo del estudio es evaluar la validez de los diagnósticos informatizados de alta hospitalarios de anomalías congénitas, comparándolos con la información contenida en la historia clínica.

Métodos: A partir de los registros de alta generados durante un año en 7 hospitales de la Comunidad Valenciana, se seleccionó aleatoriamente a 100 niños por cada hospital. Como estándar, se indicaron y codificaron los diagnósticos de las historias clínicas. Sólo se consideraron las altas generadas durante el primer año de edad. También se estudió el género, gravedad y la presentación aislada o múltiple de las anomalías congénitas. Se calculó la sensibilidad, especificidad, valores predictivos y sus intervalos de confianza al $95 \%$ por el método binomial exacto para el estudio de casos (niños) y la sensibilidad y el valor predictivo positivo para el estudio de diagnósticos.

Resultados: Se detectaron 126 niños con anomalías congénitas y 201 diagnósticos en las historias clínicas, y 83 niños con anomalías congénitas y 108 diagnósticos en los registros. Para la detección de casos los registros presentaron una sensibilidad del $64 \%$, una especificidad del $99,1 \%$ y unos valores predictivos positivos y negativos mayores del $90 \%$. En la detección de diagnósticos, la sensibilidad fue del $46 \%$ y el valor predictivo positivo del $83 \%$. La sensibilidad varió mucho según los diagnósticos.

Conclusiones: Los registros de alta hospitalaria presentan una especificidad y unos valores predictivos elevados pero una baja sensibilidad. Estos hechos se deben considerar cuando se utilicen estos registros como fuente de casos para los estudios epidemiológicos de las anomalías congénitas.

Palabras clave: Validación. Anomalías congénitas. Diagnósticos de alta hospitalaria, Sensibilidad. Especificidad. Conjunto mínimo básico de datos. Registros de alta hospitalaria.

Correspondencia:

Nieves Alba Moratilla.

Unidad de Salud Laboral. Centro de Salud Pública de Valencia. C/ San Vicente, n. ${ }^{\circ} 83,1 .^{\text {a }}$

46007 Valencia

Correo electrónico: pablo.rodriguez@ sanidad.m400.gva.es

Fax: 963602200

\section{ABSTRACT \\ Minimum Basic Data Set as a Source of Information on Congenital Defects}

Background: The purpose of this study is that of assessing the validity of the computerized diagnoses of hospital discharges of congenital defects by comparing them with the information included in the medical history.

Methods: Based on the discharge records generated over a one-year period at 7 hospitals in the Autonomous Region of Valencia, 100 children were selected at random from each hospital. As a standard, the diagnoses stated in the medical histories were indexed and coded. Solely those discharges having taken place during the first year of life were considered. A study was also made of the type, seriousness and individual or combinations of congenital defects. A calculation was made of the sensitivity, specificity, predictive values and the $95 \%$ confidence intervals thereof by the exact binomial method for the case studies (children) and the positive predictive value and sensitivity for the study of diagnoses.

Results: 126 children were detected as having congenital defects, and 201 diagnoses in medical records, and 83 children with congenital defects and 108 diagnoses on record. For the detection of cases, the records showed a $64 \%$ sensitivity, a $99.1 \%$ specificity and some positive and negative predictive values of over $90 \%$. With regard to the detection of diagnoses, the sensitivity was $46 \%$ and the positive predictive value $83 \%$. The sensitivity varied a great deal depending upon the diagnoses.

Conclusions: The hospital discharge records revealed a high degree of specificity and high predictive values, but a low degree of sensitivity. These facts must be considered when these records are used as a source of cases for the epidemiological studies of congenital defects.

Key words: Validation. Congenital defects. Hospital discharge diagnoses. Sensitivity. Specificity. Minimum Basic Set of Data. Hospital Discharge Records. 


\section{INTRODUCCIÓN}

Las anomalías congénitas (AC) se presentan aproximadamente en el $3 \%$ de todos los recién nacidos, siendo las responsables de más de las tres cuartas partes de los defectos físicos y constituyen la quinta causa de años potenciales de vida perdidos en la población '. Actualmente, el gran crecimiento industrial, la introducción en el ambiente de nuevos agentes químicos a una velocidad mayor de la necesaria para su investigación, la participación cada vez más activa de la mujer en el mundo laboral y la posibilidad de que una exposición del padre también pueda producir $\mathrm{AC}$, determinan un potencial aumento del riesgo de $\mathrm{AC}$ por factores ambientales ${ }^{2}$, lo que hace necesario el desarrollo de sistemas de información que permitan su vigilancia e investigación.

Frente a esta necesidad de información se pueden plantear dos alternativas no excluyentes: a) establecer un registro específico y b) aprovechar las fuentes de datos existentes.

En España existen diferentes registros específicos de anomalías congénitas, unos de nivel local, como el Registre de Defectes Congènits de Barcelona, el Registro de Defectos Congénitos de Asturias y el Registro del País Vasco, y otros de nivel nacional, como el «Estudio Colaborativo Español de Malformaciones Congénitas», que es de base hospitalaria y de carácter voluntario ${ }^{2}$. En la Comunidad Valenciana no existe ningún registro específico.

Fuentes potenciales de información de anomalías congénitas son los certificados de defunción, las historias de los servicios de maternidad y de neonatología, de cirugía pediátrica, los informes de autopsias, los exámenes citogenéticos, diagnósticos prenatales, los consejos genéticos o los registros de altas hospitalarias, entre otros. El uso de estas fuentes está limitado, en unas ocasiones por su poca exhaustividad, en otras por la falta de automatización de los datos y en otras por problemas de validez de los mismos.

Los registros específicos, para su puesta en marcha y mantenimiento, necesitan de importantes recursos humanos, materiales y económicos. En el caso de que sea válido, el utilizar un registro ya existente como el conjunto mínimo básico de datos (CMBD), que recoge sistemáticamente los datos de las altas hospitalarias, entre otros los diagnósticos al alta ${ }^{3}$, es más económico, al no suponer grandes costes añadidos. Este registro es una de las escasas fuentes disponibles que recoge información sobre este tipo de patologías. De hecho, en algunos países se utiliza como fuente de detección de $\mathrm{AC}^{4}$.

El objetivo del presente trabajo es evaluar la exactitud de los diagnósticos de $\mathrm{AC}$ en las altas hospitalarias según el CMBD, comparándolos con la información contenida en las historias clínicas $(\mathrm{HC})$.

\section{MATERIAL Y MÉTODOS}

La población base del estudio la componen todos los niños que, con 12 meses o menos de edad, fueron ingresados y dados de alta a lo largo de un año en siete hospitales públicos de la Comunidad Valenciana.

El cálculo de la muestra se realizó, para un intervalo de confianza del $95 \%$, suponiendo un porcentaje de casos de $\mathrm{AC}$ del $8 \% \pm 1 \%$ y una sensibilidad, en la detección de casos, del $80 \% \pm 10 \%$. El tamaño de la muestra requerida fué de 762 sujetos.

Debido a la diferente disponibilidad de datos presumiblemente fiables en cada hospital, el año de estudio fue distinto. Así, para el hospital Universitario «La Fe» de Valencia el año de estudio fuc 1992; para los hospitales generales de Elche, Orihuela, de Xátiva y Sagunto fue 1993; y para el hospital general de Castellón y Clínico de Valencia fue 1994. Para la obtención de la muestra fueron seleccionados, mediante muestreo aleatorio simple, 100 niños en cada hospital entre todas las 
altas de niños menores de un año registradas durante el año de estudio correspondiente. En total se seleccionaron 700 niños, de los cuales fueron excluidos del estudio y sustituidos por otros a través de un muestreo aleatorio simple entre las altas restantes 41 niños, por no tener codificado ningún diagnóstico al alta y 17 niños más por no haberse podido acceder a su historia clínica.

La anomalía congénita se definió como toda patología codificada según la Clasificación Internacional de Enfermedades 9. revisión, Modificación Clínica (CIE-9-MC) ${ }^{5}$ entre los códigos 740 y 759.9. En cada niño se consideraron todos los diagnósticos de $\mathrm{AC}$ registrados en el CMBD de todas las altas para ese niño habidas durante el año de estudio como si se tratara de un único episodio de alta. Estos diagnósticos se validaron comparándolos con toda la información contenida en la historia clínica hasta el último episodio de alta objeto de estudio. Los diagnósticos recogidos en la historia clínica fueron codificados de manera independiente por dos codificadores previamente entrenados, según la CIE-9-MC, con tres y cuatro dígitos. En caso de discordancia se discutían los casos hasta alcanzar un consenso. Ambos codificadores desconocían los códigos incluidos en el CMBD.

El análisis se realizó en dos niveles. En el primero se estudiaron los niños y se clasificaron como «verdaderos positivos» cuando se detectaba alguna $\mathrm{AC}$ en ambas fuentes, como «verdaderos negativos» cuando no existían en ninguna, como «falsos positivos» cuando sólo se mencionaba uno o más diagnósticos de $\mathrm{AC}$ en los registros informatizados de alta, y como «falsos negativos» cuando sólo estaban reflejados en la historia clínica. En un segundo nivel se estudiaron los diagnósticos de $\mathrm{AC}$ y se clasificaron como «verdaderos positivos» cuando coincidían todos los dígitos de un diagnóstico de $\mathrm{AC}$ en un mismo individuo en ambas fuentes; como «falsos negativos» cuando el diagnóstico sólo estaba reflejado en la historia clínica y no existía ningún diagnóstico coincidente en el CMBD; y como «falsos positivos» cuando sólo estaba presente en el CMBD.

En los dos análisis se tuvieron en cuenta las siguientes variables: género, gravedad de la anomalía congénita (menor o mayor), presentación aislada o múltiple, número de altas y hospital de procedencia. Se han calificado como anomalías congénitas menores las definidas como tal en la Guía del Programa de Promoción de la Salud Infantil editada por la Consellería de Sanitat i Consum ${ }^{6}$ de la Generalitat Valenciana y como mayores al resto.

En el análisis epidemiológico referido a los niños se calculó la sensibilidad, la especificidad y los valores predictivos positivo y negativo y sus intervalos de confianza al 95\% ( $\left(\mathrm{IC}_{95 \%}\right)$ para el conjunto de la muestra y por hospitales mediante el método binomial exacto. En el análisis de los diagnósticos se calculó la sensibilidad y el valor predictivo positivo para el total de diagnósticos, para cada diagnóstico con tres y cuatro dígitos de la CIE-9-MC y para los diagnósticos agrupados según órganos o sistemas.

\section{RESULTADOS}

El total de altas registradas en el CMBD en menores de un año en los siete hospitales objeto del estudio durante el año correspondiente a cada uno de ellos fue de 7.218 , que correspondieron a 6.265 niños, con una media de 1,15 altas por niño. Los 700 niños de la muestra causaron 820 altas, con una media de 1,17 altas por niño. El número medio de altas entre los niños que tenían $\mathrm{AC}$ fue de 1,21 y de 1,16 entre los que no las tenían. No se observó una diferencia estadísticamente significativa en la proporción de niños con anomalías congénitas en la muestra respecto al total de altas según el CMBD.

La proporción de casos con AC sobre el total de casos incluidos en el estudio, como obscrvamos en la tabla 1, varía según hospital y según la fuente de datos sea la historia clínica $(\mathrm{HC})$ o el $\mathrm{CMBD}$, siendo siempre superior en la $\mathrm{HC}$. 
Tabla 1

Número de casos de anomalías congénitas (n), proporción sobre el total de la muestra (P) e intervalo de confianza al $95 \%$ (IC95\%) según la fuente de datos y el hospital, en 700 niños menores de un año dados de alta en siete hospitales de la Comunidad Valenciana

\begin{tabular}{|lrrrrrr|}
\hline & \multicolumn{4}{c}{$C M B D$} & \multicolumn{2}{c|}{ Historia Clínica } \\
\cline { 2 - 7 } Hospitales & $n$ & $P(\%)$ & $I C(95 \%)$ & $n$ & $P(\%)$ & $I C(I C 95 \%)$ \\
\hline La Fe (Valencia) & 29 & 29,0 & $12,5-45,5$ & 35 & 35,0 & $19,2-50,8$ \\
Clínico (Valencia) & 7 & 7,0 & $0,0-25,9$ & 21 & 21,0 & $3,6-38,4$ \\
General (Castellón) & 13 & 13,0 & $0,0-31,3$ & 23 & 23,0 & $5,8-40,2$ \\
Sagunto (Valencia) & 12 & 12,0 & $0,0-30,4$ & 16 & 16,0 & $0,0-34,0$ \\
Xátiva (Valencia) & 11 & 11,0 & $0,0-29,5$ & 18 & 18,0 & $0,3-35,7$ \\
Elche (Alicante) & 6 & 6,0 & $0,0-25,0$ & 7 & 7,0 & $0,0-25,9$ \\
Orihuela (Alicante) & 5 & 5,0 & $0,0-24,1$ & 6 & 6,0 & $0,0-25,0$ \\
Totales & 83 & 11,9 & $9,5-14,3$ & 126 & 18,0 & $15,2-20,8$ \\
\hline
\end{tabular}

CMBD: Conjunto mínimo básico de datos de los registros informatizados de las altas hospitalarias.

De los 126 niños con $\mathrm{AC}$ según la historia clínica, el $71 \%(n=90)$ presentaron AC aisladas y el $75 \%(n=94)$ AC mayores. Presentaron AC mayores y aisladas el $49 \%(n=62)$ de los niños.

En un primer nivel de análisis, en el estudio de detección de niños con $\mathrm{AC}$, se observó una sensibilidad del $61,9 \%$
$\left(\mathrm{IC}_{95 \%}: 53,4-70,4\right)$ y una especificidad y valores predictivos superiores al $90 \%$. La sensibilidad varió mucho según hospitales, la menor fue del $33,3 \%$ en el hospital Clínico y la mayor del $80 \%$ del hospital la Fe. La especificidad apenas varió (tabla 2). Los índices de exactitud variaron muy poco según el género.

Tabla 2

Número de casos verdaderos positivos (VP) e Índices de exactitud del CMBD en la detección de niños con anomalías congénitas según hospitales, en $\mathbf{7 0 0}$ niños menores de un año dados de alta en siete hospitales de la Comunidad Valenciana

\begin{tabular}{|lccccc|}
\hline \multirow{2}{*}{ Hospitales } & $V P$ & \multicolumn{4}{c|}{ Indices de exactitud } \\
\cline { 5 - 7 } & & $S(\%)$ & $E(\%)$ & $V P P(\%)$ & $V P N(\%)$ \\
\hline La Fe (Valencia) & 28 & 80 & 98,5 & 96,6 & 90,1 \\
Clínico (Valencia) & 7 & 33,3 & 100 & 100 & 84,9 \\
General (Castellón) & 11 & 61,1 & 100 & 100 & 92,1 \\
Sagunto (Valencia) & 12 & 47,8 & 97,4 & 84,6 & 86,2 \\
Xátiva (Valencia) & 11 & 75,2 & 100 & 100 & 95,5 \\
Elche (Alicante) & 5 & 71,4 & 98,9 & 83,3 & 97,9 \\
Orihuela (Alicante) & 4 & 66,7 & 98,9 & 80 & 97,9 \\
Totales & 78 & 61,9 & 99,1 & 94 & 92,2 \\
\hline
\end{tabular}

CMBD: Conjunto mínimo básico de datos de los rcgistros informatizados de las altas hospitalarias; S: Sensibilidad; E: Especificidad, VPP: Valor predictivo positivo; VPN: Valor predictivo negativo.

La sensibilidad del CMBD para detectar casos varió según la gravedad o presentación de AC (tabla 3). Así, el CMBD detectó mejor las $\mathrm{AC}$ mayores y las $\mathrm{AC}$ múltiples que las $\mathrm{AC}$ menores y las $\mathrm{AC}$ aisladas. 
Tahla 3

Número de casos verdaderos positivos (VP), y sensibilidad del CMBD con el intervalo de confianza al 95\% (IC95\%) según el tipo de anomalías congénitas, en 700 niños menores de un año dados de alta en siete hospitales de la Comunidad Valenciana

\begin{tabular}{|lccc|}
\hline & $V P$ & \multicolumn{2}{c|}{ Sensibilidad } \\
\cline { 3 - 4 } Tipo de AC & $n$ & $S(\%)$ & IC (95\%) \\
\hline Casos de AC aisladas & 49 & 54,4 & $43,2-65,6$ \\
Casos de AC múltiples & 29 & 80,6 & $67,7-93,5$ \\
Casos de AC menores & 13 & 41,9 & $24,5-59,3$ \\
Casos de AC mayores & 66 & 68,1 & $58,7-77,5$ \\
\hline
\end{tabular}

CMBD: Conjunto mínimo básico de datos de los regi stros informatizados de las altas hospitalarias.
En el segundo nivel de análisis, en las historias clínicas se codificaron un total de 201 diagnósticos de $\mathrm{AC}$ y en el CMBD 112. Cuando se comparan ambas fuentes según los tres primeros dígitos de la CIE9-MC, el número de diagnósticos verdaderos positivos fue de 93 , el de falsos positivos de 19 y el de falsos negativos fue 108 (tabla 4). Por lo tanto, el CMBD, para el conjunto de diagnósticos, mostró una sensibilidad del $46,3 \%\left(\mathrm{IC}_{95 \%}\right.$ : 39,4-53,2) y un valor predictivo positivo del $83,0 \%$ $\left(\mathrm{IC}_{95 \%}: 76,1-89,9\right)$. Ambos índices variaron según hospitales (tabla 5).

\section{Tabla 4}

Distribución de los diagnósticos (CIE-9-MC, tres dígitos) de anomalías congénitas según el CMBD y la historia clínica en niños menores de un año en siete hospitales de la Comunidad Valenciana.

\begin{tabular}{|c|c|c|c|c|c|c|c|c|c|c|c|c|c|c|c|c|c|c|c|c|c|}
\hline \multicolumn{22}{|c|}{ HISTORIAS CLÍNICAS } \\
\hline CMBD & 741 & 742 & 743 & 744 & 745 & 746 & 747 & 748 & 749 & 750 & 751 & 752 & 753 & 754 & 755 & 756 & 757 & 758 & 759 & (FP) & TOTAL \\
\hline 741 & 2 & & & & & & & & & & & & & & & & & & & 0 & 2 \\
\hline 742 & & 7 & & & & & & & & & & & & & & & & & & 1 & 8 \\
\hline 743 & & & 0 & & & & & & & & & & & & & & & & & 0 & 0 \\
\hline 744 & & & & 2 & & & & & & & & & & & & & & & & 0 & 2 \\
\hline 745 & & & & & 12 & & & & & & & & & & & & & & & 1 & 13 \\
\hline 746 & & & & & & 8 & & & & & & & & & & & & & & 4 & 12 \\
\hline 747 & & & & & & & 2 & & & & & & & & & & & & & 2 & 4 \\
\hline 748 & & & & & & & & 4 & & & & & & & & & & & & 4 & 8 \\
\hline 749 & & & & & & & & & 3 & & & & & & & & & & & 2 & 5 \\
\hline 750 & & & & & & & & & & 16 & & & & & & & & & & 2 & 18 \\
\hline 751 & & & & & & & & & & & 7 & & & & & & & & & 0 & 7 \\
\hline 752 & & & & & & & & & & & & 7 & & & & & & & & 0 & 7 \\
\hline 753 & & & & & & & & & & & & & 3 & & & & & & & 0 & 3 \\
\hline 754 & & & & & & & & & & & & & & 7 & & & & & & 0 & 7 \\
\hline 755 & & & & & & & & & & & & & & & 1 & & & & & 0 & 1 \\
\hline 756 & & & & & & & & & & & & & & & & 4 & & & & 0 & 4 \\
\hline 757 & & & & & & & & & & & & & & & & & 1 & & & 1 & 2 \\
\hline 758 & & & & & & & & & & & & & & & & & & 4 & & 0 & 4 \\
\hline 759 & & & & & & & & & & & & & & & & & & & 3 & 2 & 5 \\
\hline$(\mathrm{FN})$ & 0 & 3 & 4 & 7 & 8 & 18 & 6 & 2 & 1 & 5 & 4 & 9 & 2 & 21 & 6 & 3 & 6 & 1 & 2 & - & 112 \\
\hline TOTAL & 2 & 10 & 4 & 9 & 20 & 26 & 8 & 6 & 4 & 21 & 11 & 16 & 5 & 28 & 7 & 7 & 7 & 5 & 5 & 19 & 201 \\
\hline
\end{tabular}

CIE-9-MC: 9." Revisión de la Clasificación Internacional de Enfermedades,, modificación clínica; FP: Falsos positivos; FN: Falsos negativos; CMBD: Conjunto mínimo básico de datos de los registros informatizados de las altas hospitalarias.

Como se observa en la tabla 4, las AC más frecuentes según la HC fueron «Determinadas deformidades músculo-esqueléti- cas» (código: 754), «Otras anomalías congénitas del corazón» (código: 746), «Otras anomalías congénitas de la parte superior del 


\section{Tabla 5}

Número de diagnósticos verdadero positivos (VP), Sensibilidad (S) y Valor predictivo positivo (VPP) del CMBD en cuanto la detección de diagnósticos de anomalías congénitas, distribución según hospitales, en 700 niños menores de un año dados de alta en siete hospitales de la Comunidad Valenciana

\begin{tabular}{|lccc|}
\hline Hospitales & VP & $\begin{array}{c}\text { Sensibilidad } \\
S(\%)\end{array}$ & $\begin{array}{c}\text { Valor } \\
\text { predictivo } \\
\text { positivo } \\
\text { VPP (\%) }\end{array}$ \\
\hline La Fe (Valencia) & 38 & 65,5 & 76,0 \\
Clínico (Valencia) & 7 & 18,4 & 100 \\
General (Castelión) & 12 & 33,3 & 80,0 \\
Sagunto (Valencia) & 12 & 63,2 & 100 \\
Xátiva (Valencia) & 12 & 36,4 & 100 \\
Elche (Alicante) & 8 & 72,7 & 72,7 \\
Orihuela (Alicante) & 4 & 66,7 & 80,0 \\
Totales & 93 & 46,3 & 83,0 \\
\hline
\end{tabular}

CMBD: Conjunto mínimo básico de datos de los registros informatizados de las altas hospitalarias;

aparato digestivo» (código: 750) y las «Anomalías del tronco común arterial y defectos septales» (código: 745). En conjunto representaron casi el $50 \%$ de los diagnósticos registrados. La sensibilidad del CMBD para detectar estas AC, como se puede calcular a partir de la tabla 4 , fue del $25,0 \%$, $30,8 \%, 76,2 \%$ y $60,0 \%$, respectivamente.

Considerando un nivel de desagregación de cuatro dígitos, las $\mathrm{AC}$ para las que se observó una mayor sensibilidad fueron «Estenosis hipertrófica pilórica congénita» (código: 750.5), «Defectos del tabique interventricular» (código: 745.4) y «Otras anomalías de laringe, traquea y bronquios» (código: 748.3). Por contra, los diagnósticos peor detectados fueron «Anomalías no especificadas del corazón» (código: 746.9), «Defectos del tabique interauricular tipo Ostium Secundum» (código: 745.5), «Luxación congénita de cadera» (código: 754.3) y «Ciertas deformidades músculoesqueléticas congénitas de cráneo, cara y mandíbula» (código: 754.0).

$\mathrm{Al}$ agrupar las $\mathrm{AC}$ según órganos o sistemas, observamos en la tabla 6 que las patologías mejor detectadas fueron las «Anoma- lías cromosómicas», la «Patología del sistema nervioso central», la «Fisura palatina $\mathrm{y}$ labio leporino», y la «Patología del sistema digestivo». La sensibilidad del CMBD fue mayor del $70 \%$ en todas ellas. Por contra, las patologías peor detectadas fueron las «Anomalías congénitas de oreja, cara y cuello», las «Anomalías congénitas de los tegumentos»y las «Anomalías congénitas del ojo», siendo la sensibilidad menor del 23\%. Al realizar el mismo análisis para cada hospital, cabría destacar el hecho que en el hospital La Fe, el CMBD tuvo una sensibilidad del $70,0 \%$ para detectar AC del «Corazón y sistema circulatorio» a diferencia del conjunto de hospitales cuya sensibilidad para detectar esta patología fue del $40,7 \%$.

Tabla 6

Nímero de casos de anomalías congénitas (n) en la historia clínica, número de casos verdaderos positivos (VP) y sensibilidad (S) del CMBD para detectar anomalías congénitas agrupadas según órganos o sistemas, en niños menores de un año dados de alta en siete hospitales de la Comunidad Valenciana

\begin{tabular}{|c|c|c|c|}
\hline \multirow{2}{*}{$\begin{array}{c}\text { Anomalías Congénitas } \\
\text { CIE-9-MC) }\end{array}$} & \multirow{2}{*}{$\begin{array}{c}H C \\
n\end{array}$} & \multicolumn{2}{|c|}{$C M B D$} \\
\hline & & $V P$ & $S(\%)$ \\
\hline Anomalías cromosómicas (758) & 5 & 4 & 80,0 \\
\hline Sistema nervioso central ( $740-742)$ & 12 & 9 & 75,0 \\
\hline Fisura palatina y labio leporino (749) & 4 & 3 & 75,0 \\
\hline Sistema digestivo $(750-751)$ & 32 & 23 & 71,9 \\
\hline Sistema respiratorio (748) & 6 & 4 & 66,7 \\
\hline Sistema urinario (753) & 5 & 3 & 60,0 \\
\hline $\begin{array}{l}\text { Anomalías congénitas no especifica- } \\
\text { das (759) }\end{array}$ & 5 & 3 & 60,0 \\
\hline Órganos genitales (752) & 16 & 7 & 43,8 \\
\hline $\begin{array}{l}\text { Corazón y sistema circulatorio (745- } \\
745 \text { ) }\end{array}$ & 54 & 22 & 40,7 \\
\hline $\begin{array}{l}\text { Sistema músculo-esquelético (754- } \\
756)\end{array}$ & 42 & 12 & 28,6 \\
\hline $\begin{array}{l}\text { Anomalías congénitas de la oreja, cara } \\
\text { y cuello ( } 744)\end{array}$ & 9 & 2 & 22,2 \\
\hline $\begin{array}{l}\text { Anomalías congénitas de los tegu- } \\
\text { mentos (757) }\end{array}$ & 7 & 1 & 14,3 \\
\hline Anomalías congénitas del ojo (743) & 4 & 0 & 0,0 \\
\hline Tolales $(740-759)$ & 201 & 93 & 46,3 \\
\hline
\end{tabular}

HC: Historia clínica; CMBD: Conjunto mínimo básico de datos de los registros informatizados de las altas hospitalarias. 


\section{DISCUSIÓN}

Los resultados del estudio sólo han podido ser comparados con otros dos trabajos que también analizaron la validez de los diagnósticos de alta de AC como fuentes de información, aunque con metodología distinta (7 y 8). Entre las publicaciones españolas y las internacionales no ha sido hallado ningún trabajo de este tipo.

Calle y Khoury ${ }^{7}$ compararon, en niños recién nacidos, los diagnósticos de altas hospitalarios con la información contenida en el registro médico al nacimiento que, según los autores, incluía todas las malformaciones congénitas detectadas antes del alta. En otro estudio, Hexter et al. ${ }^{8}$ compararon los diagnósticos de altas hospitalarias al nacimiento y los diagnósticos recogidos en el certificado de nacimiento con la información del sistema de vigilancia activa de anomalías congénitas de California (California Birth Defects Monitoring Program), que incluye las anomalías detectadas durante el primer año de vida.

$\mathrm{Al}$ analizar la detección de niños con $\mathrm{AC}$, los resultados del presente estudio muestran una baja sensibilidad $(62 \%)$, similar a la descrita en los trabajos de Calle $(51 \%)$ y de Hexter $(63 \%)$, y una elevada especificidad y valores predictivos, superiores al $90 \%$, a diferencia del estudio de Hexter en el que el VPP fue del $40 \%$, probablemente debida al uso de metodologías distintas.

La sensibilidad fue muy diferente en cada uno de los hospitales estudiados, destacando por su mayor sensibilidad el hospital la $\mathrm{Fe}$ de Valencia. Podría influir el hecho de ser el hospital de referencia, u otros factores como la experiencia, formación, volumen de trabajo, etcétera. Sin embargo, estas diferencias deben ser analizadas con cautela debido a que el tamaño de la muestra limita la potencia del estudio.

Según el tipo de AC, el presente estudio coincidió con el de Calle y Khoury en que la scnsibilidad para detectar niños con de- fectos mayores $(68,1 \%)$ fue más elevada que cuando los defectos eran menores $(41,9 \%)$. En el trabajo de Calle, estas sensibilidades fueron respectivamente del $72 \%$ y del $34 \%$.

En cuanto a la detección de diagnósticos, para el conjunto de los mismos, la sensibilidad del registro es del $46 \%$ y el valor predictivo positivo del $83 \%$. En el estudio realizado por Hexter et al. los valores predictivos positivos, para casos y diagnósticos, son mucho más elevados. Este autor compara diagnósticos al nacimiento con información generada durante el primer año de vida, y tal como menciona en su discusión, algunos falsos positivos podrían ser debidos a diagnósticos que se descartan con posterioridad al nacimiento. En nuestro estudio los diagnósticos de alta son comparados con toda la información contenida en la historia clínica, que incluye la generada con anterioridad al alta.

$\mathrm{Al}$ analizar la detección de diagnósticos de $\mathrm{AC}$, se observa que la sensibilidad de los registros varía mucho según grupos de defectos y/o según códigos específicos de anomalías congénitas. Al igual que en el estudio de Calle y Khoury, los registros hospitalarios son más sensibles para detectar patologías graves como las que afectan al sistema nervioso central y anomalías cromosómicas. $\mathrm{Y}$ al igual que en el estudio de Hexter, la sensibilidad para detectar AC que afecten al corazón y al sistema circulatorio es menor del $42 \%$; en el trabajo de Calle y Khoury fue muy superior $(70 \%)$, probablemente por la no inclusión de diagnósticos realizados bajo sospecha, tales como soplos sistólicos a estudio, a diferencia del presente trabajo y el de Hexter et al. La sensibilidad para detectar anomalías inespecificadas del corazón, en el que estaría incluido este tipo de patología, fue del $92 \%$ en el estudio de Calle y Khoury, del $48 \%$ en el de Hexter et al. y del $22 \%$ en el presente estudio.

La sensibilidad para detectar defectos del sistema digestivo (72\%) es mucho mayor en nuestro estudio, que en el de Hexter et al. 
Factores que pueden estar influyendo son: que en ruestro estudio se considera más de un alta por niño y que las altas consideradas son generadas durante el primer año de edad, mientras que en el estudio de Hexter et al. las altas consideradas son las ocasionadas poco después del nacimiento. Además, algunas $\mathrm{AC}$ del sistema digestivo se manifiestan meses después del nacimiento, como es el caso de la estenosis pilórica hipertrófica, cuya sensibilidad en nuestro estudio fue del $88,2 \%$ mientras que en el de Hexter et al fue del $2,1 \%$.

Los tres trabajos han coincidido en que para las anomalías de la oreja, cara y cuello, la sensibilidad es próxima al $20 \%$, y para los defectos de los tegumentos, la sensibilidad es menor del $15 \%$, estando estos grupos diagnósticos entre las patologías peor detectadas. También existe coincidencia en el hecho de que la sensibilidad para detectar fisura palatina y labio leporino es mayor del $60 \%$.

Por los comentarios expuestos consideramos que, dependiendo de la calidad del CMBD de los hospitales, este sistcma puede constituir una fuente de información válida para la detección de $\mathrm{AC}$, dependiendo de varios factores. Los índices de exactitud serán mayores cuando las $\mathrm{AC}$ sean más graves, se presente de forma múltiple y cuando se consideren en el estudio más de un alta por niño o se analicen los diagnósticos agrupados por órganos o sistemas. Una limitación del presente trabajo la constituye el número de elementos de la muestra, insuficiente para detectar AC infrecuentes.

Dependiendo de la finalidad perseguida con el conocimiento de los niños con anomalías congénitas, el registro de altas puede ser una alternativa válida $\mathrm{y}$, sobre todo, no demanda ningún esfuerzo económico y organizativo adicional.

Cuando la finalidad perseguida sea usar el CMBD como fuente de casos de AC para posteriores estudios de investigación, tipo casos y controles, la infradetección de casos podría afectar a la validez de los estudios si ésta estuviera relacionada con la exposición a estudio y/o los falsos negativos fueran incluidos como controles sin anomalías congénitas. Ambas posibilidades deben considerarse en cada caso particular. Sin embargo, dado que el valor predictivo negativo obtenido es bastante elevado (92\%), la probabilidad del segundo extremo es baja. En la validez del proceso de selección de casos de $\mathrm{AC}$ influirá también el número de altas consideradas por niño y el período de seguimiento desde el nacimiento.

Por otro lado, los registros de alta han mejorado substancialmente y lo siguen haciendo paulatinamente desde 1992 y, quizá, un análisis más actual ofreciese mejores resultados.

Destacar, por último, que el porcentaje de $\mathrm{AC}$ encontrado tanto en las historias clínicas como en el CMBD es muy superior al presumiblemente existente en la población general, investigaciones futuras deberían esclarecer si el elevado porcentaje responde a que se trata de niños hospitalizados, a que el problema está infravalorado en la población o a que el porcentaje de AC en la Comunidad Autónoma Valenciana es superior.

\section{BIBLIOGRAFÍA}

1. U.S. Preventive Task Force Services. Screening de anomalías congénitas. En: Una valoración de la efectividad de 169 intervenciones. Guía de actividades preventivas en la práctica médica. Madrid: Díaz de Santos; 1992.

2. Salvador J, Plasència A. Registro de defectos congénitos en la ciudad de Barcelona. Manual operativo. Barcelona: Ajuntament de Barcelona; 1993.

3. Boletín Oficial del Estado. Orden de 8 de octubre de 1992, de la Consellería de Sanitat i Consum, por la que se regula el conjunto mínimo básico de datos a utilizar en la información hospitalaria. DOGV núm 1891, 28/10/92.

4. De Wals P. Evaluation des risques tératogènes: l'apport des registres de malformations. Rev Epidemiol Santé Publique 1994;42:392-398.

5. Instituto Nacional de la Salud. CIE-9-MC Clasificación Internacional de Enfermedades 9." Revi- 
sión. Modificación Clínica. Volum 1. Madrid: Instituto Nacional de la Salud, 1989.

6. Consellería de Sanitat i Consum. Guía del Programa de Promoción de la Salud Infantil. Valencia: Consellería de Sanitat i Consum; 1991.

7. Calle EE, Khoury MJ. Completeness of the discharge diagnoses as a measure of birth defects recorded in the hospital birth record. Am J Epidemiol 1991;134:69-77.

8. Hexter AC, Harris JA, Roeper P, Croen LA, Krueger $P$, Gant $D$. Evaluation of the hospital discharge diagnoses index and the birth certificate as sources of information on birth defects. Public Health Rep 1990; 105: 296-307. 